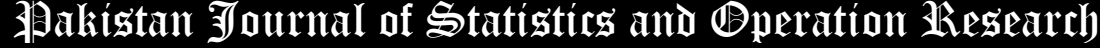

\section{An Interpretation of Non-Preemptive Priority Fuzzy Queuing Model with Asymmetrical Service Rates}

\author{
K.Usha Prameela ${ }^{* 1}$, R. Richard Wurmbrand ${ }^{2}$, R P S Jayakar ${ }^{3}$ \\ * Corresponding Author \\ 1. K L University, Vijayawada, India, kushaprameela@gmail.com \\ 2. Osmania University, Hyderabad, India, rwrwurmbrand@gmail.com \\ 3. Geethanjali College, Hyderabad, India, peterrregulagadda@gmail.com
}

\begin{abstract}
This paper presents Non-Preemptive priority fuzzy queuing model with asymmetrical service rates. Arrival rate and service rate are taken to be hexagonal, heptagonal, and octagonal fuzzy numbers. Here an interpretation is given to determine the performance measures by applying a new ranking technique through which the fuzzy values are reduced to the crisp values. This ranking technique has the benefit of being precise and relevant compared to other methods such as alpha-cut method and LR method. The main intention is to evaluate the fuzziness before the performance measures are processed by utilizing the regular queueing hypothesis. Three numerical examples are exhibited to show the validity implementation of the methodology.
\end{abstract}

Key Words: Priority queuing model, hexagonal fuzzy number ,heptagonal fuzzy number, and octagonal fuzzy number, new ranking technique.

\section{Introduction}

These days, the idea of queuing hypothesis has numerous applications in the real time processes. On the whole, the priority queue has a wide scope of utilizations like communication networks, transport area, medical service executives, service industry, production etc. Meanwhile, the idea of fuzzy queues is broadly discussed by scientists such as (by Yager. (1981)), (by Li. and Lee.(1989)), (by Negi. and Lee. (1992)), (by Kao. and Wilson.(1999)), (by Chen. (2005)), (by Wagner. (2010)), (by Hajipour. (2014)), (by Mueen. (2017)), (by Aria. (2019)), who fostered the idea of fuzzy queues. Earlier extensive research has been conducted on Queueing systems with priority customers. The monograph of (by Jaiswal.(1968)) provided an excellent unified account of queueing systems under priority disciplines. (by McMillan. (1995)) specified the utilization of priority queueing frameworks in cell versatile organizations. (by Choi. and Chang. (1999)) proposed several models such as telephone in the restaurant, supporter line modules of phone trades, communication conventions and channel allocation scheme in wireless networks. (by Pardo. and Fuente.(2007)) optimized a priority discipline queuing model using fuzzy set theory.

There are two possible segments in priority situation, the Preemption and Non-Preemption. (by Yeo. (1963)) analysed Preemptive priority queues with K classes of customers with a Preemptive repeat and a Preemptive resume strategy. (by Chang. (1965)) proposed a single server queueing system with Non-Preemptive and Preemptive resume priorities. (by Miller.(1981)) obtained steady-state distributions of exponential single server (Preemptive and Non-Preemptive) priority queues with two classes of customers by using Transient analysis of a Non-Preemptive Neut's theory of matrix-geometric invariant probability vectors. (by Brandt. and Brandt. (2004)) contemplated a two-class M/M/1 queueing system under Preemptive resume and impatience of the prioritized customers. Non-Preemptive priority fuzzy queues have been studied (by Devaraj. and Jayalakshmi. (2012)) where fuzzy problem is reduced to crisp problem. 
(by Palpandi. and Geetharamani. (2013)) computed performance measures of fuzzy Non-Preemptive priority queues by Robust ranking technique. The above overview shows that the analysis of Non-Preemptive priority fuzzy queueing systems has not been studied in many cases. Thus, in this paper, an approach to deal with an interpretation of a NonPreemptive priority queueing system in fuzzy environment with asymmetrical service rates is given.

This paper is coordinated as follows: Section 2 describes some basic definitions, Section 3 presents methodology, section 4 clarifies fuzzy queuing model, Section 5 depicts the plan of new ranking technique, Section 6 presents mathematical interpretation by taking numerical examples, Section 7 gives the results and discussion, section 8 finishes the article with future directions.

\section{Essential ideas and definitions}

The essential ideas and definitions are given as follows

Fuzzy set (by Zadeh.1965)): Let X be a classical set or a universe. A fuzzy subset $\bar{D}$ (or a fuzzy set $\bar{D}$ ) in $\mathrm{X}$ is defined by the function $\mu \bar{D}$, called membership function of $\bar{D}$, from X to the real unit interval $[0,1] . \mu \bar{D}$ (a) is called the grade or the membership degree of a, $\forall \mathrm{a} \in \bar{D}$.

Fuzzy number (by Zadeh.(1965)): A fuzzy set $\bar{D}$ characterized on the set of real numbers R is said to be a fuzzy number, if $\bar{D}$ has the accompanying qualities such as

1. $\bar{D}$ is normal, i.e., $\sup \mu_{\bar{D}}(\mathrm{x})=1$.

2. $\bar{D}$ is convex set, i.e.,

$$
\mu_{\bar{D}}(\lambda x+(1-\lambda) y) \geq \min \left(\mu_{\bar{D}}(\mathrm{x}), \mu_{\bar{D}}(\mathrm{y})\right), \forall \mathrm{x}, \mathrm{y} \in \mathrm{X}, \forall \lambda \in[0,1] .
$$

Hexagonal fuzzy number(HXFN) (by Zimmerman . (1985)) : A fuzzy number $\bar{D}$ is a Hexagonal fuzzy number characterized by $\left(d_{1}, d_{2}, d_{3}, d_{4}, d_{5}, d_{6}\right)$ where $d_{1}, d_{2}, d_{3}, d_{4}, d_{5}, d_{6}$ are real numbers. Its membership function is given below.

$$
\mu_{\bar{D}}(x)=\left\{\begin{array}{cc}
0 & \text { for } \quad x<d_{1} \\
\frac{1}{2}\left(\frac{x-d_{1}}{d_{2}-d_{1}}\right) & \text { for } d_{1} \leq \mathrm{x} \leq d_{2} \\
\frac{1}{2}+\frac{1}{2}\left(\frac{x-d_{2}}{d_{3}-d_{2}}\right) & \text { for } d_{2} \leq \mathrm{x} \leq d_{3} \\
1 & \text { for } d_{3} \leq \mathrm{x} \leq d_{4} \\
1-\frac{1}{2}\left(\frac{x-d_{4}}{d_{5}-d_{4}}\right) & \text { for } d_{4} \leq \mathrm{x} \leq d_{5} \\
\frac{1}{2}\left(\frac{d_{6}-x}{d_{6}-d_{5}}\right) & \text { for } d_{5} \leq \mathrm{x} \leq d_{6} \\
0 & \text { otherwise }
\end{array}\right.
$$

(i) $\quad d_{1}$ to $d_{5}$ is a monotonically increasing continuous real-valued function.

(ii) $\quad \mathrm{d}_{5}$ to $\mathrm{d}_{6}$ is monotonically decreasing continuous real-valued function

(iii) $\quad \mathrm{d}_{1} \leq \mathrm{d}_{2} \leq \mathrm{d}_{3} \leq \mathrm{d}_{4} \leq \mathrm{d}_{5} \leq \mathrm{d}_{6}$.

Heptagonal fuzzy number(HPTN) (by Zimmerman. (1985)) : A fuzzy number $\bar{D}$ is a Heptagonal fuzzy number characterized by $\left(\mathrm{d}_{1}, \mathrm{~d}_{2}, \mathrm{~d}_{3}, \mathrm{~d}_{4}, \mathrm{~d}_{5}, \mathrm{~d}_{6}, \mathrm{~d}_{7}\right)$ where $\mathrm{d}_{1}, \mathrm{~d}_{2}, \mathrm{~d}_{3}, \mathrm{~d}_{4}, \mathrm{~d}_{5}, \mathrm{~d}_{6}, \mathrm{~d}_{7}$ are real numbers. Its membership function is given below. 


$$
\mu_{\bar{D}}(x)=\left\{\begin{array}{cc}
0 & \text { for } \quad x<d_{1} \\
\frac{1}{2}\left(\frac{x-d_{1}}{d_{2}-d_{1}}\right) & \text { for } d_{1} \leq \mathrm{x} \leq d_{2} \\
\frac{1}{2}+\frac{1}{2}\left(\frac{x-d_{2}}{d_{3}-d_{2}}\right) & \text { for } d_{2} \leq \mathrm{x} \leq d_{3} \\
1 & \text { for } d_{3} \leq \mathrm{x} \leq d_{4} \\
1-\frac{1}{4}\left(\frac{x-d_{4}}{d_{5}-d_{4}}\right) & \text { for } d_{4} \leq \mathrm{x} \leq d_{5} \\
\frac{3}{4}-\frac{1}{2}\left(\frac{x-d_{5}}{d_{6}-d_{5}}\right) & \text { for } d_{5} \leq \mathrm{x} \leq d_{6} \\
\frac{1}{4}\left(\frac{x-d_{7}}{d_{6}-d_{7}}\right) & \text { for } d_{6} \leq \mathrm{x} \leq d_{7} \\
0 & x>d_{7}
\end{array}\right.
$$

(i) $\quad d_{1}$ to $d_{7}$ is a monotonically increasing continuous real-valued function.

(ii) $\quad \mathrm{d}_{1} \leq \mathrm{d}_{2} \leq \mathrm{d}_{3} \leq \mathrm{d}_{4} \leq \mathrm{d}_{5} \leq \mathrm{d}_{6} \leq \mathrm{d}_{7}$.

Octagonal fuzzy number (OCFN) (by Zimmerman. (1985)): A fuzzy number $\bar{D}$ is an Octagonal fuzzy number characterized by $\left(\mathrm{d}_{1}, \mathrm{~d}_{2}, \mathrm{~d}_{3}, \mathrm{~d}_{4}, \mathrm{~d}_{5}, \mathrm{~d}_{6}, \mathrm{~d}_{7}, \mathrm{~d}_{8}\right)$ where $\mathrm{d}_{1}, \mathrm{~d}_{2}, \mathrm{~d}_{3}, \mathrm{~d}_{4}, \mathrm{~d}_{5}, \mathrm{~d}_{6}, \mathrm{~d}_{7}, \mathrm{~d}_{8}$ are real numbers. Its membership function is given below.

$$
\mu_{\bar{D}}(x)=\left\{\begin{array}{cc}
0 & \text { for } \quad x<d_{1} \\
k\left(\frac{x-d_{1}}{d_{2}-d_{1}}\right) & \text { for } d_{1} \leq \mathrm{x} \leq d_{2} \\
k & \text { for } d_{2} \leq \mathrm{x} \leq d_{3} \\
k+(1-k)\left(\frac{x-d_{3}}{d_{4}-d_{3}}\right) & \text { for } d_{3} \leq \mathrm{x} \leq d_{4} \\
1 & \text { for } d_{4} \leq \mathrm{x} \leq d_{5} \\
k+(1-k)\left(\frac{d_{6}-x}{d_{6}-d_{5}}\right) & \text { for } d_{5} \leq \mathrm{x} \leq d_{6} \\
k & \text { for } d_{6} \leq \mathrm{x} \leq d_{7} \\
\frac{1}{4}\left(\frac{d_{8}-x}{d_{8}-d_{7}}\right) & \text { for } d_{7} \leq \mathrm{x} \leq d_{8} \\
0 & x>d_{8}
\end{array}\right.
$$

(i) $\quad \mathrm{d}_{1}$ to $\mathrm{d}_{8}$ is a monotonically increasing continuous real-valued function.

(ii) $\quad \mathrm{d}_{1} \leq \mathrm{d}_{2} \leq \mathrm{d}_{3} \leq \mathrm{d}_{4} \leq \mathrm{d}_{5} \leq \mathrm{d}_{6} \leq \mathrm{d}_{7} \leq \mathrm{d}_{8}$.

\section{Methodology}

In this section we provide a solution methodology for the proposed model i.e. Non-Preemptive priority fuzzy queuing model. The crisp values of the fuzzy arrival rate and the fuzzy service rate were determined by new ranking method. The main purpose is to determine the performance measures by utilizing the regular queueing hypothesis . In this model the service rates are asymmetrical. Here fuzziness is evaluated before the performance measures are processed.

\section{Model description}

\section{Non-Preemptive priority fuzzy queue with asymmetrical service rates}

Let us consider a single server two-class Non-Preemptive priority queue with various service rates. The inter arrival rate of $\lambda_{1}$ and $\lambda_{2}$ are appropriated independently. The service rates $\mu_{1}$ and $\mu_{2}$ are additionally circulated independently. FCFS line discipline is followed, though the low priority customer gets prior service than the high priority customers. From the classical queueing hypothesis, 


$$
\begin{aligned}
& L_{\mathrm{q}}{ }^{(1)}=\frac{\lambda 1\left(\frac{\lambda 1}{\mu 1^{\wedge} 2}+\frac{\lambda 2}{\mu 2^{\wedge} 2}\right)}{1-\rho 1} \\
& L_{\mathrm{q}}{ }^{(2)}=\frac{\lambda 2\left(\frac{\rho 1}{\mu 1}+\frac{\rho 2}{\mu 2}\right)}{(1-\rho 1)((1-\rho)}
\end{aligned}
$$

The stability steady state is $\rho \equiv \rho_{1}+\rho_{2}<1$, essential, $0<\rho<1$. Where $\rho_{1}=\frac{\lambda \mathbf{1}}{\mu \mathbf{1}}, \quad \rho 2=\frac{\lambda 2}{\mu 2}$

Other exhibition estimations are characterized by:

$$
\begin{gathered}
\mathrm{W}_{\mathrm{q}}^{(\mathrm{i})}=\frac{\mathbf{L q}}{\lambda i} \\
\mathrm{~W}_{\mathrm{s}}^{(i)}=\mathrm{Wq}^{(i)}+\frac{1}{\mu i} \\
\mathrm{~L}_{\mathrm{s}}^{(\mathrm{i})}=\lambda_{\mathrm{i}} \mathrm{Ws}^{(\mathrm{i})} ; \quad \mathrm{i}=1,2
\end{gathered}
$$

\section{Proposed New Ranking Technique}

To change the fuzzy values into real crisp values, the accompanying new ranking technique mentioned below is utilized.

$$
R(\tilde{F})=\frac{(\mathrm{d} \min +\mathrm{d} \max )}{2}
$$

whereas $\mathrm{d}_{\min }$ and $\mathrm{d}_{\max }$ are the minimum and maximum values of the given fuzzy number.

\section{Mathematical Interpretation}

Let us now assume a critical situation that happens in a corporate hospital in Hyderabad during covid 19 time, where some corona patients with breathing difficulty have arrived in need of medical treatment. In such emergency, the doctor allows the patients to immediately receive the treatment (Non-Preemptive priority only). The average queue length and average waiting time of that two-class Non-Preemptive priority corona patients queue on this possibility is currently computed.

\subsection{Hexagonal Fuzzy Number}

Let $\lambda_{1}=[1,3,5,7,9,11]$ and $\lambda_{2}=[2,4,6,8,10,12]$ are the arrival rates and $\mu_{1}=[21,23,25,27,29,31]$ and $\mu_{2}=[22$, $24,26,28,30,32]$ are two different service rates respectively.

According to equation (11)

$$
\begin{aligned}
& R\left(\lambda_{1}\right)=(1+11) / 2=6 \\
& R\left(\lambda_{2}\right)=(2+12) / 2=7 \\
& R\left(\mu_{1}\right)=(21+31) / 2=26 \\
& R\left(\mu_{2}\right)=(22+32) / 2=27 \\
& \rho_{1}=\lambda_{1} / \mu_{1}=0.2307 \\
& \rho_{2}=\lambda_{2} / \mu_{2}=0.2592 \\
& \rho=\rho_{1}+\rho_{2}=0.4899
\end{aligned}
$$

From equations (6), (7), (8), (9) \& (10) the performance measures are determined and is presented in Table 1. 
Table 1: Performance Measures- $L_{\mathrm{q}}, L_{\mathrm{s}} \&_{W \mathrm{~s}}, \mathrm{~W}_{\mathrm{q}}$

\begin{tabular}{lllll}
\hline $\begin{array}{l}\text { Number of } \\
\text { priorities }\end{array}$ & $(\mathbf{L q})$ & $\left(\mathbf{L s}_{\mathbf{s}}\right)$ & $(\mathbf{W q})$ & $\left(\mathbf{W}_{\mathbf{s}}\right)$ \\
\hline $\begin{array}{l}\text { First } \\
\text { priority }\end{array}$ & 0.1441 & 0.3744 & 0.024 & 0.0624 \\
$\begin{array}{l}\text { Second } \\
\text { priority }\end{array}$ & 0.3295 & 0.588 & 0.0470 & 0.0840 \\
\hline
\end{tabular}

Avg. queue length (Lq),Avg. system length $\left(\mathrm{L}_{\mathrm{s}}\right)$

Avg. waiting time in queue (Wq), Avg. waiting time in system (Ws),

\subsection{Heptagonal Fuzzy Number}

Let $\lambda 1=[10,12,14,16,18,20,22]$ and $\lambda 2=[11,13,15,17,19,21,23]$ are the arrival rate and $\mu 1=$ $[31,33,35,37,39,41,43]$ and $\mu 2=[32,34,36,38,40,42,44]$ are two different service rates respectively.

According to equation (11)

$$
\begin{aligned}
& R\left(\lambda_{1}\right)=(10+22) / 2=16 \\
& R\left(\lambda_{2}\right)=(11+23) / 2=17 \\
& R\left(\mu_{1}\right)=(31+43) / 2=37 \\
& R\left(\mu_{2}\right)=(32+44) / 2=38 \\
& \rho_{1}=\lambda_{1} / \mu_{1}=0.4324 \\
& \rho_{2}=\lambda_{2} / \mu_{2}=0.4473 \\
& \rho=\rho_{1}+\rho_{2}=0.8797
\end{aligned}
$$

\begin{tabular}{|c|c|c|c|c|}
\hline $\begin{array}{l}\text { Number of } \\
\text { priorities }\end{array}$ & $(\mathbf{L q})$ & (Ls) & $(\mathbf{W q})$ & $\left(\mathbf{W}_{\mathbf{s}}\right)$ \\
\hline $\begin{array}{l}\text { First } \\
\text { priority }\end{array}$ & 0.6613 & 1.0928 & 0.0413 & 0.0683 \\
\hline $\begin{array}{l}\text { Second } \\
\text { priority }\end{array}$ & 5.8401 & 6.2866 & 0.3435 & 0.3698 \\
\hline
\end{tabular}

From equations (6), (7), (8), (9) \& (10) the performance measures are determined and is presented in Table 2.

Table 2: Performance Measures - $L_{\mathrm{q}}, L_{\mathrm{s}} \&_{W_{\mathrm{s}}}, \mathbf{W}_{\mathbf{q}}$.

Avg. queue length (Lq),Avg. system length $\left(\mathrm{L}_{\mathrm{s}}\right)$

Avg. waiting time in queue (Wq), Avg. waiting time in system (Ws),

\subsection{Octagonal Fuzzy Number}

Let $\lambda 1=[20,21,22,23,24,25,26,27]$ and $\lambda 2=[28,29,30,31,32,33,34,35]$ are the arrival rate and $\mu 1=$ $[55,56,57,58,59,60,61,62]$ and $\mu 2=[63,64,65,66,67,68,69,70]$ are two different service rates respectively.

According to equation (11)

$$
\begin{gathered}
R\left(\lambda_{1}\right)=(20+27) / 2=23.5 \\
R\left(\lambda_{2}\right)=(28+35) / 2=31.5
\end{gathered}
$$




$$
\begin{aligned}
& R\left(\mu_{1}\right)=(55+62) / 2=58.5 \\
& R\left(\mu_{2}\right)=63+70 / 2=66.5 \\
& \rho_{1}=\lambda_{1} / \mu_{1}=0.4017 \\
& \rho_{2}=\lambda_{2} / \mu_{2}=0.4736 \\
& \rho=\rho_{1}+\rho_{2}=0.8753
\end{aligned}
$$

From equations (6), (7), (8), (9) \& (10) the performance measures are determined and is presented in Table 3.

Table 3: Performance Measures- - $L_{\mathrm{q}}, L_{\mathrm{s}} \& W_{\mathrm{s}}, \mathrm{W}_{\mathrm{q}}$

\begin{tabular}{lllll}
\hline $\begin{array}{l}\text { Number of } \\
\text { priorities }\end{array}$ & $(\mathbf{L q})$ & $\left(\mathbf{L s}_{\mathbf{s}}\right)$ & $(\mathbf{W q})$ & $\left(\mathbf{W}_{\mathbf{s}}\right)$ \\
\hline $\begin{array}{l}\text { First } \\
\text { priority }\end{array}$ & 2.7059 & 3.104 & 0.1151 & 0.1321 \\
$\begin{array}{l}\text { Second } \\
\text { priority }\end{array}$ & 5.9060 & 6.3756 & 0.1874 & 0.2024 \\
\hline
\end{tabular}

Avg. queue length (Lq),Avg. system length $\left(\mathrm{L}_{\mathrm{s}}\right)$

Avg. waiting time in queue (Wq), Avg. waiting time in system (Ws)

\section{Results and Discussion}

The obtained outcomes are given in Tables $1-3$, which clarify various estimations of each priority class for a wide range of membership functions considered (hexagonal, heptagonal, and octagonal fuzzy numbers). It is likewise observed from Tables that all the performance measures of first priority class is less than the performance measures of second priority class in the framework for all the three sorts of above-mentioned fuzzy numbers.

\section{Conclusions and future directions}

This paper interprets the average queue length and average waiting time of two-class Non-Preemptive priority queue with asymmetric service rates. The fuzzy non-preemptive priority queue is represented more precisely and the scientific outcomes are derived by new ranking technique. Numerical examples for hexagonal, heptagonal and octagonal fuzzy numbers are disclosed viably to decide the validity of the proposed queuing model.The crisp values of the fuzzy arrival rate and the fuzzy service rate were determined by new ranking method. It is more efficient than other existing ranking method. The future work can be done in evaluating the potency of this new ranking technique to other queueing models and other types of linear membership functions. This paper can be extended by considering the probabilistic parameter in place of fuzzy numbers. Another possible area for future research work is to consider intuitionistic fuzzy numbers and neutrosophic sets. The authors are right now working on more complex examples of customer-server associations including multiple serving channels and/or stages, just as cases for which more than one server processes a customer all the while.

\section{References}

1. Yeo, G.F. (1963). Preemptive priority queues. Journal of the Australian Mathematical Society, 3, 491-502.

2. Chang, W.(1965). Pre-emptive Priority Queues. Operations Research, 13, 1020-1022 .

3. Miller, D.R.(1981). Computation of steady-state probabilities for $\mathrm{M} / \mathrm{M} / 1$ priority queues. Operations Research, 29, 945-948 .

4. Brandt. and Brandt, M.(2004).On the two-class M/M/1 system under pre-emptive resume and impatience of the prioritized customers. Queueing Systems, 47, 147-168.

5. Kalpana, B., \& Anusheela, N. (2018). Analysis of a Single Server Non-Pre-emptive Fuzzy Priority Queue using LR Method. ARPN Journal of Engineering and Applied Sciences, 13(23), 9306-9310. 
6. Ramli, N., \& Mohamad, D. (2009). A comparative analysis of centroid methods in ranking fuzzy numbers. European Journal of Scientific Research, 28(3), 492-501.

7. Ritha, W., \& Robert, L. (2010). Fuzzy queues with priority discipline. Applied Mathematical Sciences, 4(12), 575-582.

8. Ramesh, R., \& Ghuru, S.K. (2014). Priority disciplined queuing models with fuzzy. Journal of Engineering and Applied Sciences, 4(3).

9. Kalpana, NA.B.(2019).Analysis of Fuzzy Priority Queue with two classes of customers and unequal service rates using Mixed Integer Non-Linear Programming Approach. Journal Engineering and Applied Sciences 14(15), 5030-5038.

10. McMillan, D.(1995). Delay analysis of a cellular mobile priority queueing system. IEEE/ACM Transactions on Networking, 3, 310-319.

11. Choi, B.D., and Chang, Y.(1999).Single server retrial queues with priority calls. Mathematical and Computer Modelling, 30, 7-32.

12. Ng, C-H., and Soong, B-H. (2008).Queueing Modelling Fundamentals: With Applications in Communication Networks. John Wiley \& Sons.

13. Jaiswal, N.K. (1968). Priority queues. Academic Press.

14. Aria, M. (2019). New Fuzzy Logic System for Controlling Multiple Traffic Intersections with Dynamic Phase Selection and Pedestrian Crossing Signal. Journal of Engineering. Science and Technology (JESTEC), 14(4), 1974 - 1983.

15. Wagner, C., and Hagras, H. (2010). Uncertainty and type-2 fuzzy sets and systems. Proceedings of the IEEE 10th UK Workshop on Computational Intelligence (UKCI). Colchester. United Kingdom, 1-5.

16. Hajipour, V., Khodakarami, V., and Tavana, M. (2014). The redundancy queuing-location-allocation problem: a novel approach. IEEE Transactions on Engineering Management, 61(3), 534-544.

17. Yager, R.R. (1981). A Procedure for Ordering Fuzzy Subsets of the Unit Interval. Information Science, 24(2), 143-161.

18. Mueen, Z., Ramli, R.,\& Zabidi, NZ. (2017). Analysis of performance measures with single channel fuzzy queues under two class by ranking method. Journal of Telecommunication, Electronic and Computer Engineering, 9(1-2), 109-112.

19. Selvakumaria, K., and Revathi, S.(2021). Analysis of Fuzzy Non-pre-emptive Priority Queuing Model with Unequal Service Rate. Turkish Journal of Computer and Mathematics Education, 12(5), 1457-1460.

20. Li, R.J., and Lee, E.S, (1989). Analysis of fuzzy queues. Computers and Mathematics with Applications, 17(7), 1143-1147.

21. Negi.D.S., and Lee. E. S. (1992). Analysis and Simulation of Fuzzy Queue,. Fuzzy Sets and Systems, .46, 321-330.

22. Kao, C., Li, C.C., and Chen, S.P. (1999). Parametric Programming to the Analysis of Fuzzy Queue. Fuzzy Sets and Systems, 107(1), 93-100.

23. Chen.S. P. (2005). Parametric Nonlinear Programming Approach to Fuzzy Queues with Bulk Service. European Journal of Operations Research, vol 163, No.2, 434 - 444.

24. Zadeh, L.A . (1965). Fuzzy Sets. Information and Control, vol. 8, pp. 338-353.

25. Zimmermann, H. J. (2011). Fuzzy Set Theory and its Applications. (4th, Ed.) New York: Springer Science and Business Media.

26. Pardo, M.J., and David de la Fuente. (2007). Optimizing a priority discipline queuing model using fuzzy set theory. Computers and Mathematics with Applications, 54: 267281.

27. Palpandi, B., and Geetharamani, G. (2013). Computing Performance Measures of Fuzzy Non-Preemptive Priority Queues Using Robust Ranking Technique. Applied Mathematical Analysis, 7(102), 5095-5102.

28. Devaraj. and Jayalakshmi, D. ( 2012). A Fuzzy Approach to Priority Queues. International Journal of Fuzzy Mathematics and System, 2(4),479-488. 28. 